\title{
Low adiponectin concentration during pregnancy predicts postpartum insulin resistance, beta cell dysfunction and fasting glycaemia
}

\author{
R. Retnakaran • Y. Qi • P. W. Connelly • M. Sermer • \\ A. J. Hanley • B. Zinman
}

Received: 14 July 2009 /Accepted: 7 October 2009/Published online: 24 November 2009

(C) Springer-Verlag 2009

\begin{abstract}
Aims/hypothesis The postpartum phase following gestational diabetes (GDM) is characterised by subtle metabolic defects, including the beta cell dysfunction that is believed to mediate the increased future risk of type 2 diabetes in this patient population. Low circulating levels of adiponectin and increased leptin and C-reactive protein (CRP) have recently emerged as novel diabetic risk factors, although their relevance to GDM and subsequent diabetes has not been characterised. Thus, we sought to determine whether adiponectin, leptin and CRP levels during pregnancy relate to the postpartum metabolic defects linking GDM with type 2 diabetes.
\end{abstract}

Electronic supplementary material The online version of this article (doi:10.1007/s00125-009-1600-8) contains supplementary material, which is available to authorised users.

R. Retnakaran $(\bowtie) \cdot$ Y. Qi $\cdot$ A. J. Hanley $\cdot$ B. Zinman

Leadership Sinai Centre for Diabetes, Mount Sinai Hospital,

60 Murray Street, Suite-L5-039, Mailbox-21, Toronto, ON,

Canada, M5T3L9

e-mail: rretnakaran@mtsinai.on.ca

R. Retnakaran $\cdot$ P. W. Connelly $\cdot$ A. J. Hanley $\cdot$ B. Zinman

Division of Endocrinology, University of Toronto,

Toronto, ON, Canada

P. W. Connelly

Keenan Research Centre, Li Ka Shing Knowledge Institute of St.

Michael's Hospital,

Toronto, ON, Canada

M. Sermer

Division of Obstetrics and Gynecology, Mount Sinai Hospital, Toronto, ON, Canada

A. J. Hanley

Department of Nutritional Sciences, University of Toronto,

Toronto, ON, Canada
Methods Metabolic characterisation, including oral glucose tolerance testing, was undertaken in 487 women during pregnancy and at 3 months postpartum. Based on the antepartum OGTT, there were 137 women with GDM, 91 with gestational impaired glucose tolerance and 259 with normal glucose tolerance.

Results Adiponectin levels were lowest $(p<0.0001)$ and CRP levels highest $(p=0.0008)$ in women with GDM. Leptin did not differ between the glucose tolerance groups $(p=0.4483)$. Adiponectin $(r=0.41, p<0.0001)$, leptin $(r=-0.36, p<0.0001)$ and CRP $(r=-0.30, p<0.0001)$ during pregnancy were all associated with postpartum insulin sensitivity (determined using the insulin sensitivity index of Matsuda and DeFronzo [IS $\left.\mathrm{SGTT}_{\mathrm{OGT}}\right]$ ). Intriguingly, adiponectin levels were also related to postpartum beta cell function (insulinogenic index/HOMA of insulin resistance; $r=0.16, p=0.0009$ ). Indeed, on multiple linear regression analyses, adiponectin levels during pregnancy independently predicted both postpartum insulin sensitivity $(t=3.97$, $p<0.0001)$ and beta cell function $(t=2.37, p=0.0181)$, even after adjustment for GDM. Furthermore, adiponectin emerged as a significant negative independent determinant of postpartum fasting glucose $(t=-3.01, p=0.0027)$.

Conclusions/interpretation Hypoadiponectinaemia during pregnancy predicts postpartum insulin resistance, beta cell dysfunction and fasting glycaemia, and hence may be relevant to the pathophysiology relating GDM with type 2 diabetes.

Keywords Adiponectin - Beta cell function - CRP. Gestational diabetes $\cdot$ Leptin $\cdot$ Type 2 diabetes
Abbreviations
CRP C-reactive protein
GDM Gestational diabetes 


$\begin{array}{ll}\text { GIGT } & \text { Gestational impaired glucose tolerance } \\ \text { HOMA-IR } & \text { HOMA of insulin resistance } \\ \text { IGI } & \text { Insulinogenic index } \\ \text { IS } & \text { Insulin sensitivity index of Matsuda and } \\ & \text { DeFronzo } \\ \text { NDDG } & \text { National Diabetes Data Group } \\ \text { NGT } & \text { Normal glucose tolerance }\end{array}$

\section{Introduction}

The diagnosis of gestational diabetes mellitus (GDM) identifies a population of young women at high risk of subsequently developing type 2 diabetes [1,2]. As such, it is not surprising that detailed postpartum evaluation of women with a history of GDM typically reveals subtle metabolic defects, including the pathological hallmarks of type 2 diabetes: chronic insulin resistance and beta cell dysfunction [1]. The chronic beta cell defect, in particular, is believed to play a central role in determining the future risk of type 2 diabetes [1,3]. Importantly, however, the precise pathophysiological mediators of postpartum metabolic dysfunction and diabetic risk in this patient population remain to be fully elucidated.

Identifying specific metabolic intermediates during pregnancy that are associated with future risk of type 2 diabetes mellitus may provide insight into the pathophysiology underlying this relationship. Antepartum factors that have been linked to the future development of type 2 diabetes in women with GDM include: pre-pregnancy BMI; the severity of hyperglycaemia at diagnosis; earlier gestational age at diagnosis; recurrent GDM; parity; and non-white ethnicity [1, 4-6]. Of these, pre-pregnancy BMI appears to be particularly important, having emerged as the factor with the highest attributable risk fraction [6]. Thus, obesitymediated factors may be relevant to the pathophysiological relationship between GDM and subsequent type 2 diabetes.

In the past decade, a growing body of evidence has identified two pathological sequelae of obesity that may link adiposity to diabetic risk: (1) chronic subclinical inflammation, as characterised by increased serum levels of inflammatory biomarkers such as C-reactive protein (CRP); and (2) dysregulation of adipokines, including increased serum leptin and low circulating levels of the insulin-sensitising protein adiponectin [7, 8]. Indeed, CRP, leptin and hypoadiponectinaemia have each emerged as novel risk factors for type 2 diabetes mellitus in the general population [8-11]. Considering these data, we hypothesised that these factors may also be relevant to GDM and subsequent type 2 diabetes, a setting in which their role has not been characterised. Thus, our objective in this study was to determine whether CRP, leptin and adiponectin during pregnancy relate to postpartum insulin resistance, beta cell dysfunction and glycaemia in a cohort of women representing the full spectrum of glucose homeostasis during pregnancy, ranging from normal through impaired glucose tolerance to GDM.

\section{Methods}

This analysis was conducted in the context of an ongoing observational study of early events in the natural history of type 2 diabetes, in which a cohort of women recruited at the time of antepartum screening for GDM is undergoing longitudinal metabolic characterisation during pregnancy and the postpartum period [12-14]. The study protocol, which was approved by the Mount Sinai Hospital Research Ethics Board, has been described in detail previously [12-14] and all participants have provided written informed consent. In brief, participants have undergone: (1) a $3 \mathrm{~h}$ $100 \mathrm{~g}$ OGTT in late second trimester for determination of glucose tolerance status during pregnancy; and (2) a $2 \mathrm{~h}$ $75 \mathrm{~g}$ OGTT at 3 months postpartum, with completion of interviewer-administered questionnaires, a physical examination and assessment of cardio-metabolic risk factors on both occasions [12-14].

Laboratory measurements and physiological indices All OGTTs were performed in the morning after an overnight fast, with venous blood samples drawn for measurement of glucose and insulin at fasting and at 30,60 and $120 \mathrm{~min}$ (and 180 min during pregnancy) following ingestion of the glucose load. As previously described [12, 14], the application of National Diabetes Data Group (NDDG) criteria [15] to the antepartum OGTT stratified participants into the following three glucose tolerance groups during pregnancy: (1) GDM (defined by exceeding two or more NDDG glycaemic thresholds); (2) gestational impaired glucose tolerance (GIGT) (a designation that was not originally described by NDDG but that we have previously applied to describe those women exceeding only one NDDG glycaemic threshold on the OGTT); and (3) normal glucose tolerance (NGT).

Specific insulin was measured using the Roche Elecsys 1010 immunoassay analyser and the electrochemiluminescence immunoassay kit (Roche Diagnostics, Laval, Quebec, Canada). This assay shows $0.05 \%$ cross-reactivity with intact human proinsulin and the primary circulating split form (des 31, 32). Total adiponectin was measured from fasting serum samples by enzyme-linked immunosorbent assay (Linco, St Louis, Missouri, USA). High-sensitivity CRP was measured by endpoint nephelometry using the Dade-Behring BN Prospec and the N High Sensitivity CRP reagent (Dade Behring, Mississauga, ON, Canada). Leptin 
was measured using the human leptin ELISA assay \#EZHL80SK (Linco Research, St Charles, Missouri, USA).

Insulin sensitivity was measured using the insulin sensitivity index of Matsuda and DeFronzo (IS OGTT $_{\text {) [16]. }}$ The HOMA of insulin resistance (HOMA-IR) was calculated as described by Matthews et al. [17]. Beta cell function was assessed by the insulinogenic index divided by HOMA-IR (insulinogenic index/HOMA-IR) $[18,19]$.

Statistical analyses All analyses were conducted using the Statistical Analysis System (SAS, version 9.1, SAS Institute, Cary, NC, USA). Continuous variables were tested for normality of distribution and natural log transformations of skewed variables were used, where necessary, in subsequent analyses. In Table 1, univariate differences across the three gestational glucose tolerance groups were assessed during pregnancy and at 3 months postpartum using ANOVA for continuous variables and $\chi^{2}$ test for categorical variables. Univariate correlations between metabolic variables during pregnancy and at 3 months postpartum were assessed by Spearman correlation analysis (Table 2). Multiple linear regression analysis (Table 3) was used to determine independent relationships between factors during pregnancy and the following dependent variables at 3 months postpartum: $\log \mathrm{IS}_{\text {OGTT }}$ (model $\mathrm{A}$ ), $\log$ insulinogenic/HOMA-IR (model B), and log fasting glucose (model $\mathrm{C}$ ). The antepartum variables that were included in these models consisted of: (1) those that have been linked to future diabetic risk in pregnant women (age, weeks' gestation, pre-pregnancy BMI, gestational weight gain preceding the OGTT, ethnicity, family history of diabetes, personal history of previous GDM, parity, GIGT in current pregnancy, GDM in current pregnancy); and (2) the biochemical factors under study (CRP, leptin, adiponectin). In these models, the reference groups were white for ethnicity and nulliparous for parity, respectively.

Table 1 Characteristics of study participants stratified by glucose tolerance status during pregnancy

\begin{tabular}{|c|c|c|c|c|}
\hline Characteristic & NGT $(n=259)$ & GIGT $(n=91)$ & $\operatorname{GDM}(n=137)$ & $p$ value \\
\hline \multicolumn{5}{|l|}{ At OGTT during pregnancy } \\
\hline Age (years) & $33.9(4.3)$ & $34.2(4.2)$ & $34.5(4.3)$ & 0.3606 \\
\hline Weeks gestation (weeks) & $30(28-32)$ & $29(28-31)$ & $29(28-31)$ & 0.0002 \\
\hline Ethnicity & & & & 0.0835 \\
\hline White $(\%)$ & 79.5 & 71.4 & 71.5 & \\
\hline Asian $(\%)$ & 8.5 & 19.8 & 11.0 & \\
\hline Other $(\%)$ & 12.0 & 8.8 & 17.5 & \\
\hline Pre-pregnancy BMI $\left(\mathrm{kg} / \mathrm{m}^{2}\right)$ & $23.1(21.3-26.9)$ & $23.5(21.8-27.7)$ & $25.0(22.0-30.1)$ & 0.0114 \\
\hline Weight gain during pregnancy $(\mathrm{kg})$ & $11.4(8.6-14.5)$ & $10.0(7.3-14.5)$ & $9.1(5.9-12.7)$ & 0.0019 \\
\hline Family history of DM (\%) & 47.5 & 52.8 & 59.1 & 0.0271 \\
\hline Previous GDM/macrosomia (\%) & 2.3 & 12.1 & 7.3 & 0.0144 \\
\hline Parity & & & & 0.3978 \\
\hline Nulliparous (\%) & 46.7 & 55.3 & 50.4 & \\
\hline One or greater $(\%)$ & 53.3 & 44.7 & 49.6 & \\
\hline CRP (mg/l) & $4.7(2.6-7.5)$ & $4.1(2.4-8.6)$ & $5.8(3.5-10.7)$ & 0.0008 \\
\hline Leptin (ng/ml) & $32.9(23.0-49.1)$ & $35.7(23.3-47.6)$ & $35.8(25.4-43.4)$ & 0.4483 \\
\hline Adiponectin $(\mu \mathrm{g} / \mathrm{ml})$ & $8.0(6.2-10.0)$ & $7.0(5.2-8.7)$ & $7.0(5.3-8.5)$ & $<0.0001$ \\
\hline \multicolumn{5}{|l|}{ At 3 months postpartum } \\
\hline Breastfeeding (\%) & 93.1 & 87.9 & 95.6 & 0.5131 \\
\hline BMI $\left(\mathrm{kg} / \mathrm{m}^{2}\right)$ & $25.4(23.0-28.9)$ & $26.0(23.2-30.1)$ & $26.6(23.7-31.1)$ & 0.0701 \\
\hline CRP (mg/l) & $1.6(0.9-3.6)$ & $2.5(1.0-5.4)$ & $2.7(1.5-4.9)$ & 0.0003 \\
\hline Leptin (ng/ml) & $16.8(8.4-30.9)$ & $23.9(11.6-35.3)$ & $23.8(13.0-38.7)$ & 0.0006 \\
\hline Adiponectin $(\mu \mathrm{g} / \mathrm{ml})$ & $8.6(6.6-10.6)$ & $7.6(5.4-9.9)$ & $8.2(6.1-10.4)$ & 0.0463 \\
\hline IS $_{\text {OGTT }}$ & $11.9(8.0-16.9)$ & $8.9(6.0-12.6)$ & $8.5(5.7-12.5)$ & $<0.0001$ \\
\hline Insulinogenic index/HOMA-IR & $11.4(7.5-17.4)$ & $8.9(5.2-12.4)$ & $8.1(4.4-12.3)$ & $<0.0001$ \\
\hline Fasting glucose $(\mathrm{mmol} / \mathrm{l})$ & $4.4(4.2-4.7)$ & $4.7(4.4-5.0)$ & $4.7(4.4-5.0)$ & $<0.0001$ \\
\hline
\end{tabular}

Data are presented as median followed by interquartile range, with the exception of: (1) age (presented as mean followed by standard deviation); and (2) ethnicity, family history of diabetes mellitus, previous GDM, parity and breastfeeding (presented as percentages)

$p$ values refer to overall differences across groups as derived from ANOVA for continuous variables or $\chi^{2}$ test for categorical variables 
Table 2 Spearman univariate correlations between: (1) adiponectin, CRP, and leptin during pregnancy and at 3 months postpartum; and (2) insulin sensitivity (IS OGTT $_{\text {), beta cell }}$ function (insulinogenic index [IGI]/HOMA-IR) and fasting glucose at 3 months postpartum

\begin{tabular}{|c|c|c|c|c|c|c|}
\hline \multirow[t]{2}{*}{ Variable } & \multicolumn{2}{|c|}{ IS $_{\text {OGTT }}$} & \multicolumn{2}{|c|}{ IGI/HOMA-IR } & \multicolumn{2}{|c|}{ Fasting glucose } \\
\hline & $r$ & $p$ value & $r$ & $p$ value & $r$ & $p$ value \\
\hline \multicolumn{7}{|c|}{ At 3 months postpartum } \\
\hline CRP & -0.39 & $<0.0001$ & -0.05 & 0.31 & 0.22 & $<0.0001$ \\
\hline Leptin & -0.59 & $<0.0001$ & -0.13 & 0.007 & 0.43 & $<0.0001$ \\
\hline Adiponectin & 0.47 & $<0.0001$ & 0.12 & 0.01 & -0.25 & $<0.0001$ \\
\hline \multicolumn{7}{|c|}{ During pregnancy } \\
\hline CRP & -0.30 & $<0.0001$ & -0.05 & 0.24 & 0.14 & 0.0021 \\
\hline Leptin & -0.36 & $<0.0001$ & -0.03 & 0.51 & 0.23 & $<0.0001$ \\
\hline Adiponectin & 0.41 & $<0.0001$ & 0.16 & 0.0009 & -0.31 & $<0.0001$ \\
\hline
\end{tabular}

\section{Results}

Characteristics of study groups during pregnancy and at 3 months postpartum Table 1 shows the baseline demographic, clinical and metabolic characteristics of the study population during pregnancy, stratified into the following three gestational glucose tolerance groups: NGT $(n=259)$, GIGT $(n=91)$, and GDM $(n=137)$. There were no significant differences between the groups with respect to age, ethnicity and parity. The antepartum OGTT was performed slightly later in the women with NGT (median 30 weeks' gestation) than in the other two groups (both median 29 weeks; overall $p=0.002)$. As glucose tolerance status worsened, both family history of diabetes and personal history of previous GDM were more prevalent ( $p=0.0271$ and $p=0.0144$, respectively). Similarly, prepregnancy BMI progressively increased with worsening glucose tolerance status $(p=0.0114)$, varying from median $23.1 \mathrm{~kg} / \mathrm{m}^{2}$ in the NGT group to $23.5 \mathrm{~kg} / \mathrm{m}^{2}$ in GIGT to $25.0 \mathrm{~kg} / \mathrm{m}^{2}$ in the GDM group. Gestational weight gain preceding the OGTT showed the opposite pattern, being greatest in NGT (most likely reflecting the later OGTT in this group, as gestational weight gain did not differ between the three groups after adjustment for weeks' gestation at the time of the OGTT $[p=0.1967])$. Of note, leptin concentration during pregnancy did not differ significantly between the three groups $(p=0.4483)$, whereas CRP levels were highest $(p=0.0008)$ and adiponectin levels lowest $(p<0.0001)$ in the women with GDM. These group differences in CRP and adiponectin concentration also persisted after adjustment for weeks' gestation at the time of the OGTT (Electronic supplementary material [ESM] Table 1).

At 3 months postpartum, there remained significant metabolic differences between the groups (Table 1). Specifically, as expected, both insulin sensitivity $\left(\right.$ IS $\left._{\text {OGTT }}\right)$ and beta cell function (insulinogenic index/HOMA-IR) progressively decreased from the NGT group to GIGT to GDM (both $p<0.0001$ ), while fasting glucose showed the opposite pattern (being lowest in NGT) $(p<0.0001)$. Furthermore, the
NGT group exhibited the highest adiponectin concentration (overall $p=0.0463$ ) and the lowest CRP and leptin levels (overall $p=0.0003$ and $p=0.0006$, respectively).

Relationships between metabolic variables during pregnancy and those at 3 months postpartum CRP, leptin and adiponectin levels during pregnancy were each strongly correlated with their respective concentrations at 3 months postpartum (CRP: $r=0.60, p<0.0001$; leptin: $r=0.71$, $p<0.0001$; adiponectin: $r=0.78, p<0.0001)$. When measured at 3 months postpartum, these proteins were crosssectionally associated with variables of glucose homeostasis (Table 2). Specifically, CRP and leptin were both positively associated with fasting glucose and inversely related to IS OGTT, while adiponectin showed the opposite relationships (inverse with fasting glucose and positive with IS $_{\text {OGTT }}$ ). In addition, postpartum leptin was negatively associated with insulinogenic index/HOMA-IR, while adiponectin was positively related.

As CRP, leptin and adiponectin during pregnancy were strongly correlated with their respective levels at 3 months postpartum, which in turn were associated with variables of glucose homeostasis at that time, we queried whether the antepartum concentrations of these proteins related to postpartum metabolic function. Indeed, on Spearman univariate correlation analysis, adiponectin, leptin and CRP during pregnancy were all associated with postpartum IS OGTT $(r=0.41, r=-0.36$, and $r=-0.30$, respectively; all $p<0.0001$; Table 2). Intriguingly, adiponectin was also related to postpartum insulinogenic index/HOMA-IR $(r=0.16, p=0.0009)$. Furthermore, adiponectin during pregnancy was more strongly associated with postpartum fasting glucose $(r=-0.31, p<0.0001)$ than were antepartum levels of either leptin $(r=0.23, p<0.0001)$ or CRP $(r=0.14$, $p=0.0021)$. Thus, on univariate analysis, adiponectin during pregnancy was positively associated with postpartum insulin sensitivity and beta cell function, and inversely associated with postpartum fasting glycaemia (Fig. 1). 
Table 3 Multiple linear regression analyses of the relationships between antepartum variables and the following variables of glucose homeostasis at 3 months postpartum: (model A) log IS $_{\text {OGTT; (model B) }}$ $\log$ IGI/HOMA-IR; and (model C) log fasting glucose
Note that reference groups are: (1) white for ethnicity; and (2) nulliparous for parity, respectively

\begin{tabular}{|c|c|c|c|c|}
\hline & Beta coefficient & Standard error & $t$ & $p$ value \\
\hline \multicolumn{5}{|l|}{ Model A } \\
\hline Age & -0.001 & 0.006 & -0.17 & 0.8643 \\
\hline Weeks' gestation & 0.020 & 0.010 & 2.03 & 0.0427 \\
\hline Pre-pregnancy BMI & -0.032 & 0.007 & -4.57 & $<0.0001$ \\
\hline Weight gain up to OGTT & -0.003 & 0.004 & -0.74 & 0.4575 \\
\hline \multicolumn{5}{|l|}{ Ethnicity } \\
\hline Asian & -0.322 & 0.084 & -3.84 & 0.0001 \\
\hline Other non-white & -0.197 & 0.084 & -2.35 & 0.0194 \\
\hline Family history of diabetes & -0.025 & 0.052 & -0.48 & 0.6345 \\
\hline Parity & 0.025 & 0.053 & 0.47 & 0.6361 \\
\hline Previous GDM & -0.176 & 0.133 & -1.32 & 0.1878 \\
\hline GIGT in current pregnancy & -0.130 & 0.069 & -1.90 & 0.0587 \\
\hline GDM in current pregnancy & -0.123 & 0.062 & -1.99 & 0.0475 \\
\hline CRP & -0.006 & 0.004 & -1.41 & 0.1600 \\
\hline Leptin & -0.003 & 0.001 & -1.78 & 0.0758 \\
\hline Adiponectin & 0.038 & 0.010 & 3.97 & $<0.0001$ \\
\hline \multicolumn{5}{|l|}{ Model B } \\
\hline Age & -0.010 & 0.010 & -1.02 & 0.3094 \\
\hline Weeks' gestation & 0.047 & 0.016 & 2.93 & 0.0036 \\
\hline Pre-pregnancy BMI & -0.020 & 0.011 & -1.75 & 0.0810 \\
\hline Weight gain up to OGTT & -0.008 & 0.007 & -1.09 & 0.2778 \\
\hline \multicolumn{5}{|l|}{ Ethnicity } \\
\hline Asian & 0.142 & 0.138 & 1.03 & 0.3025 \\
\hline Other non-white & 0.173 & 0.141 & 1.23 & 0.2211 \\
\hline Family history of diabetes & -0.112 & 0.085 & -1.33 & 0.1852 \\
\hline Parity & 0.177 & 0.087 & 2.03 & 0.0431 \\
\hline Previous GDM & -0.107 & 0.214 & -0.50 & 0.6177 \\
\hline GIGT in current pregnancy & -0.342 & 0.113 & -3.02 & 0.0027 \\
\hline GDM in current pregnancy & -0.294 & 0.101 & -2.92 & 0.0037 \\
\hline CRP & -0.011 & 0.007 & -1.73 & 0.0843 \\
\hline Leptin & 0.007 & 0.002 & 2.98 & 0.0031 \\
\hline Adiponectin & 0.037 & 0.016 & 2.37 & 0.0181 \\
\hline \multicolumn{5}{|l|}{ Model C } \\
\hline Age & 0.003 & 0.001 & 2.47 & 0.0139 \\
\hline Weeks' gestation & -0.005 & 0.002 & -2.52 & 0.0123 \\
\hline Pre-pregnancy BMI & 0.006 & 0.001 & 4.63 & $<0.0001$ \\
\hline Weight gain up to OGTT & 0.002 & 0.001 & 2.51 & 0.0123 \\
\hline \multicolumn{5}{|l|}{ Ethnicity } \\
\hline Asian & 0.023 & 0.016 & 1.47 & 0.1416 \\
\hline Other non-white & 0.029 & 0.015 & 1.92 & 0.0553 \\
\hline Family history of diabetes & -0.008 & 0.010 & -0.81 & 0.4192 \\
\hline Parity & -0.010 & 0.010 & -1.02 & 0.3078 \\
\hline Previous GDM & 0.008 & 0.025 & 0.32 & 0.7489 \\
\hline GIGT in current pregnancy & 0.047 & 0.013 & 3.65 & 0.0003 \\
\hline GDM in current pregnancy & 0.042 & 0.012 & 3.68 & 0.0003 \\
\hline $\mathrm{CRP}$ & -0.001 & 0.001 & -1.46 & 0.1459 \\
\hline Leptin & -0.00003 & 0.0003 & -0.14 & 0.8872 \\
\hline Adiponectin & -0.005 & 0.002 & -3.01 & 0.0027 \\
\hline
\end{tabular}




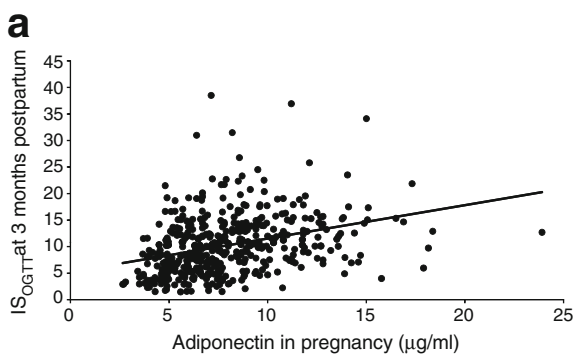

b

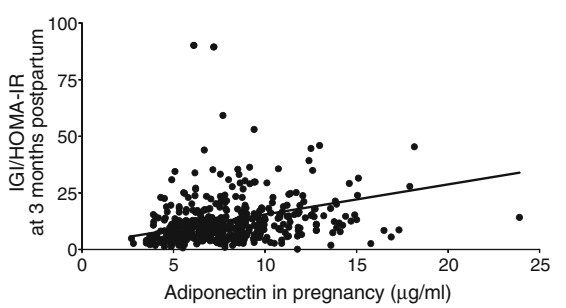

C

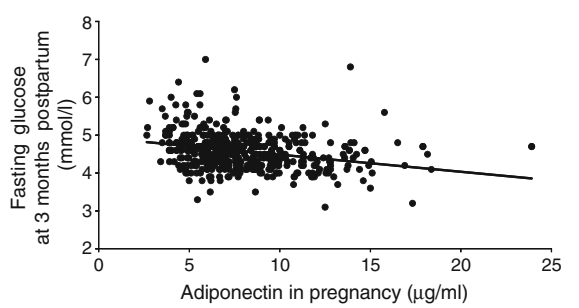

Fig. 1 Plots of relationships between adiponectin during pregnancy and the following variables of glucose homeostasis at 3 months postpartum: (a) $\operatorname{IS}_{\mathrm{OGTT}}(r=0.41, p<0.0001)$; (b) insulinogenic index/
HOMA-IR ( $r=0.16, p=0.0009)$ (note: a single outlying point with IGI/HOMA-IR 230.77 is not shown on the plot but was included in the analyses); and (c) fasting glucose $(r=-0.31, p<0.0001)$
Multiple linear regression analyses Having demonstrated that GDM is characterised by antepartum hypoadiponectinaemia, which in turn relates to postpartum insulin resistance, beta cell dysfunction and fasting glycaemia, we next queried whether adiponectin levels during pregnancy account for the postpartum metabolic dysfunction observed in women with GDM. In this context, multiple linear regression analyses (Table 3) were performed to elucidate independent relationships between antepartum variables and each of the following three postpartum outcome measures: (model A) insulin sensitivity $\left(\log\right.$ IS $_{\text {OGTT }}$ ); (model B) beta cell function (log insulinogenic index/ HOMA-IR); and (model C) glycaemia (log fasting glucose). The following antepartum variables were included in all of these models: age, weeks' gestation, pre-pregnancy BMI, gestational weight gain preceding the OGTT, ethnicity, family history of diabetes, personal history of previous GDM, parity, GIGT in the current pregnancy, GDM in the current pregnancy, CRP, leptin and adiponectin. Importantly, after adjustment for all of these variables (including notably GDM and GIGT), adiponectin during pregnancy emerged as a significant independent determinant of both postpartum insulin sensitivity $(t=3.97, p<0.0001$; Table 3 model A) and postpartum beta cell function $(t=2.37$, $p=0.0181$; Table 3 model B). Furthermore, unlike CRP and leptin during pregnancy, antepartum adiponectin emerged as a significant negative independent determinant of postpartum fasting glucose $(t=-3.01, p=0.0027$; Table 3 model C). Thus, hypoadiponectinaemia during pregnancy, as occurs in GDM, is an independent predictor of postpartum insulin resistance, beta cell dysfunction and fasting glycaemia.

Subgroup and sensitivity analyses The multiple linear regression analyses in Table 3 were repeated with stratification of participants into subgroups based on pre-pregnancy BMI and gestational glucose tolerance status. Amongst lean women (defined by pre-pregnancy BMI $<25 \mathrm{~kg} / \mathrm{m}^{2} ; n=288$ ), adiponectin remained a significant predictor of postpartum insulin sensitivity $(t=2.56, p=0.0111)$, beta cell function $(t=2.23, p=0.0269)$ and fasting glucose $(t=-2.19$, $p=0.0298$ ). In the overweight/obese group (defined by prepregnancy $B M I \geq 25 \mathrm{~kg} / \mathrm{m}^{2}$ ), adiponectin was a significant predictor of postpartum insulin sensitivity $(t=3.19, p=0.0018)$ and fasting glucose $(t=-2.37, p=0.0192)$, but not beta cell function ( $t=0.92, p=0.3599$ ). The last of these findings may be partly the result of power limitations with the smaller sample size of the overweight/obese group $(n=185)$, particularly as no significant interaction was detected between adiponectin and BMI group (lean vs overweight/obese) with respect to postpartum beta cell function $(p=0.5674$ for interaction term) (data not shown).

We next repeated the multiple linear regression analyses within the gestational glucose tolerance groups. Amongst women with NGT $(n=259)$, adiponectin remained a significant independent predictor of postpartum insulin sensitivity ( $t=2.34, p=0.0204)$, beta cell function $(t=2.03$, $p=0.0438)$ and fasting glycaemia $(t=-2.38, p=0.0182)$. These relationships were less robust in the smaller subsets of GIGT and GDM. Amongst women with GIGT $(n=91)$, adiponectin maintained significance as an independent predictor of postpartum beta cell function only $(t=2.30$, $p=0.0250)$. Amongst women with GDM $(n=137)$, adiponectin maintained significance as an independent predictor of postpartum insulin sensitivity only $(t=2.79, p=0.0064)$. Importantly, formal tests of interaction between adiponectin and gestational glucose tolerance group in the Table 3 models showed no significant interactions $(p=0.3398$, $p=0.4688$ and $p=0.9595$ for interaction terms in the insulin sensitivity, beta cell function and fasting glycaemia models, respectively; data not shown). As such, the differences observed in the GIGT and GDM groups (compared with the NGT group) are likely to reflect their much smaller sample sizes.

Finally, while the relationship between adiponectin and postpartum insulin sensitivity in Table 3 model A could be attenuated by further adjustment for IS I $_{\text {OGTT }}$ during pregnancy ( $t=1.30, p=0.1948)$, the significant independent relationship between antepartum adiponectin and postpartum beta cell function in Table 3 model B persisted even 
after adjustment for insulinogenic index/HOMA-IR during pregnancy $(t=2.11, p=0.0357$; data not shown).

\section{Discussion}

In this report, we demonstrate that GDM is characterised by increased CRP concentration and low levels of adiponectin during pregnancy and in the postpartum period. Importantly, multiple linear regression analyses reveal that antepartum hypoadiponectinaemia predicts postpartum insulin resistance, beta cell dysfunction and fasting glycaemia. These findings thus implicate adiponectin deficiency as a potential factor in the pathophysiology linking GDM with the subsequent development of type 2 diabetes, providing insight which may be relevant to both risk stratification and modification in this patient population.

Previous studies have demonstrated that, during pregnancy, women with GDM exhibit evidence of subclinical inflammation and dysregulation of adipokines, including low circulating levels of both adiponectin and its highmolecular-weight multimeric form [20-23]. In fact, both subclinical inflammation and hypoadiponectinaemia may be chronic defects in this patient population, as increased CRP and low adiponectin in the first trimester have each been shown to independently predict the subsequent development of GDM later during pregnancy $[22,23]$. Moreover, a limited number of studies following pregnancy have also reported increased levels of inflammatory markers and decreased adiponectin in women with a history of GDM [24-26]. By documenting their existence both during pregnancy and at 3 months postpartum, the current study supports the potential chronic nature of these defects, and thereby suggests that they may be of pathophysiological relevance to diabetic risk in this patient population.

In this context, the key finding of the current study is the demonstration that hypoadiponectinaemia during pregnancy is an independent predictor of postpartum metabolic dysfunction, including insulin resistance and beta cell dysfunction. Indeed, as chronic beta cell dysfunction is believed to mediate the considerable long-term risk of type 2 diabetes in women with a history of GDM [1, 3], these data suggest that hypoadiponectinaemia may be a pivotal abnormality linking these conditions. This possibility is further supported by the demonstration that, unlike dysregulation of CRP and leptin, low adiponectin during pregnancy is an independent determinant of fasting glucose at 3 months postpartum.

Several lines of evidence support the plausibility of a role for adiponectin in the relationship between GDM and type 2 diabetes. First, although not previously studied in women with a history GDM, hypoadiponectinaemia has emerged as a robust predictor of incident type 2 diabetes in a variety of settings and populations [11]. Second, low adiponectin has been repeatedly associated with insulin resistance, consistent with the known insulin-sensitising bio-activity of the protein $[8,11]$. This inverse relationship between adiponectin and insulin resistance has also been consistently observed during pregnancy and in GDM [20, $21,27]$. Finally, a growing body of literature has recently queried a link between adiponectin and beta cell function, although conclusive resolution of this question remains elusive owing to some conflicting observations in this asyet modest literature. Specifically, adiponectin receptors are expressed on beta cells [28, 29], although in vitro studies have reported conflicting findings with respect to the ability of adiponectin to stimulate insulin secretion [29, 30]. In humans, low circulating adiponectin concentration has been associated with beta cell dysfunction in some [21, 31-34], but not all [29,35], studies, possibly because of differences in: (1) the methods used to evaluate beta cell function; (2) the populations under study; and (3) their sample sizes. Of note, the only two previous studies examining this question during pregnancy have indeed linked hypoadiponectinaemia and beta cell dysfunction in women with and without GDM [21, 31]. Thus, in relating antepartum hypoadiponectinaemia to postpartum beta cell dysfunction, the current study extends these earlier data and highlights the need for further longitudinal study of this relationship.

The significance of the current findings rests in the potential implications that a relationship between antepartum adiponectin and future type 2 diabetes could hold for the stratification and modification of diabetic risk. Specifically, it follows from these data that antepartum adiponectin concentration may provide a means of stratifying women with GDM with respect to their future risk of type 2 diabetes. Ideally, this information could help to target postpartum surveillance efforts to those women at the highest risk of developing diabetes. The availability of this predictor at the time of diagnosis during pregnancy may be particularly important, in light of the well-recognised suboptimal rates of postpartum metabolic follow-up in women with GDM [36]. Second, the current data also suggest that chronic hypoadiponectinaemia could provide a therapeutic target for risk modification in this patient population. In this respect, it is of interest to note that thiazolidinedione therapy, which has been shown to preserve beta cell function and significantly reduce the risk of developing type 2 diabetes in women with a history of GDM [37], is also known to increase adiponectin levels [38]. It thus emerges that a pathophysiological relationship between hypoadiponectinaemia and diabetic risk following GDM could hold important clinical implications.

A limitation of this study is the use of surrogate (rather than direct) measures of insulin resistance and beta cell function. However, direct measures (such as clamp studies) 
would be difficult to implement in a study of this size $(n=487)$ given their cost, invasiveness and time requirements, which may be particularly burdensome for new mothers at 3 months postpartum. Moreover, both the IS OGTT $_{\text {index and insulino- }}$ genic index/HOMA-IR have been widely used in large clinical studies [19]. A second limitation is that the observational nature of these data cannot conclusively establish causality in the relationship between antepartum adiponectin and postpartum metabolic dysfunction. Nevertheless, the identification of adiponectin in this context represents a biochemical advance on our existing clinical understanding of the link between GDM and subsequent type 2 diabetes, and should lead to further study.

In summary, GDM is characterised by increased CRP concentration and low levels of adiponectin during pregnancy and in the postpartum. Importantly, antepartum hypoadiponectinaemia predicts postpartum insulin resistance, beta cell dysfunction and fasting glycaemia. Thus, adiponectin deficiency may be a factor in the pathophysiology linking GDM with the subsequent development of type 2 diabetes, and hence may be relevant to strategies for both risk stratification and risk modification in this setting.

Acknowledgements We wish to thank Mount Sinai Hospital Department of Pathology and Laboratory Medicine and Patient Care Services. This study was supported by operating grants MOP 67063 and 84206 from the Canadian Institutes of Health Research (CIHR), and OG-3-08-2543-RR from the Canadian Diabetes Association (CDA). R. Retnakaran holds a CIHR New Investigator Award, CDA Clinician-Scientist incentive funding, and University of Toronto Banting and Best Diabetes Centre New Investigator funding. A. J. Hanley holds a Tier-II Canada Research Chair in Diabetes Epidemiology. B. Zinman holds the Sam and Judy Pencer Family Chair in Diabetes Research at Mount Sinai Hospital and University of Toronto.

Duality of interest The authors declare that there is no duality of interest associated with this manuscript.

\section{References}

1. Buchanan TA, Xiang AH (2005) Gestational diabetes mellitus. J Clin Invest 115:485-491

2. Feig DS, Zinman B, Wang X, Hux JE (2008) Risk of development of diabetes mellitus after diagnosis of gestational diabetes. CMAJ 179:229-234

3. Buchanan TA (2001) Pancreatic $\beta$-cell defects in gestational diabetes: implications for the pathogenesis and prevention of type 2 diabetes. J Clin Endocrinol Metab 86:989-993

4. Schaefer-Graf UM, Buchanan TA, Xiang AH, Peters RK, Kjos SL (2002) Clinical predictors for a high risk for the development of diabetes mellitus in the early puerperium in women with recent gestational diabetes mellitus. Am J Obstet Gynecol 186:751-756

5. Sinha B, Brydon P, Taylor RS et al (2003) Maternal ante-natal parameters as predictors of persistent postnatal glucose intolerance: a comparative study between Afro-Caribbeans, Asians and Caucasians. Diabet Med 20:382-386
6. Albareda M, Caballero A, Badell G et al (2003) Diabetes and abnormal glucose tolerance in women with previous gestational diabetes. Diabetes Care 26:1199-1205

7. Pickup JC, Crook MA (1998) Is type 2 DM a disease of the innate immune system? Diabetologia 41:1241-1248

8. Rasouli N, Kern PA (2008) Adipocytokines and the metabolic complications of obesity. J Clin Endocrinol Metab 93:S64S73

9. Pradham AD, Manson JE, Rifai N, Buring J, Ridker PM (2001) C-reactive protein, interleukin 6 and risk of developing type 2 diabetes mellitus. JAMA 286:327-334

10. Welsh P, Murray HM, Buckley BM et al (2009) Leptin predicts diabetes but not cardiovascular disease: results from a large prospective study in an elderly population. Diabetes Care 32:308310

11. Li S, Shin HJ, Ding EL, van Dam RM (2009) Adiponectin levels and risk of type 2 diabetes: a systematic review and meta-analysis. JAMA 302:179-188

12. Retnakaran R, Qi Y, Sermer M, Connelly PW, Hanley AJ, Zinman B (2008) Glucose intolerance in pregnancy and future risk of prediabetes or diabetes. Diabetes Care 31:2026-2031

13. Retnakaran R, Qi Y, Sermer M, Connelly PW, Zinman B, Hanley AJ (2008) Isolated hyperglycemia at 1-hour on oral glucose tolerance test in pregnancy resembles gestational diabetes in predicting postpartum metabolic dysfunction. Diabetes Care 31:1275-1281

14. Retnakaran R, Qi Y, Sermer M, Connelly PW, Hanley AJ, Zinman B (2009) The antepartum glucose values that predict neonatal macrosomia differ from those that predict postpartum prediabetes or diabetes: implications for the diagnostic criteria for gestational diabetes. J Clin Endocrinol Metab 94:840-845

15. National Diabetes Data Group (1979) Classification and diagnosis of diabetes mellitus and other categories of glucose intolerance. Diabetes 28:1039-1057

16. Matsuda M, DeFronzo R (1999) Insulin sensitivity indices obtained from oral glucose tolerance testing: comparison with the euglycemic insulin clamp. Diabetes Care 22:1462-1470

17. Matthews DR, Hosker JP, Rudenski AS, Naylor BA, Treacher DF, Turner RC (1985) Homeostasis model assessment: insulin resistance and $\beta$-cell function from fasting plasma glucose and insulin concentrations in man. Diabetologia 28:412-419

18. Wareham NJ, Phillips DI, Byrne CD, Hales CN (1995) The 30 minute insulin incremental response in an oral glucose tolerance test as a measure of insulin secretion. Diabet Med 12:931

19. Kahn SE (2003) The relative contributions of insulin resistance and beta cell dysfunction to the pathophysiology of type 2 diabetes. Diabetologia 46:3-19

20. Retnakaran R, Hanley AJ, Raif N, Connelly PW, Sermer M, Zinman B (2004) Reduced adiponectin concentration in women with gestational diabetes: a potential factor in progression to type 2 diabetes. Diabetes Care 27:799-800

21. Retnakaran R, Connelly PW, Maguire G, Sermer M, Zinman B, Hanley AJ (2007) Decreased high molecular weight adiponectin in gestational diabetes: implications for the pathophysiology of type 2 diabetes. Diabet Med 24:245-252

22. Wolf M, Sandler L, Hsu K, Vossen-Smirnakis K, Ecker JL, Thadhani R (2003) First-trimester C-reactive protein and subsequent gestational diabetes. Diabetes Care 26:819-824

23. Williams MA, Qiu C, Muy-Rivera M, Vadachkoria S, Song T, Luthy DA (2004) Plasma adiponectin concentrations in early pregnancy and subsequent risk of gestational diabetes mellitus. J Clin Endocrinol Metab 89:2306-2311

24. Winzer C, Wagner O, Festa A et al (2004) Plasma adiponectin, insulin sensitivity, and subclinical inflammation in women with prior gestational diabetes mellitus. Diabetes Care 27:1721-1727 
25. Di Benedetto A, Russo GT, Corrado F et al (2005) Inflammatory markers in women with a recent history of gestational diabetes mellitus. J Endocrinol Investig 28:34-38

26. Di Cianni G, Lencioni C, Volpe L et al (2007) C-reactive protein and metabolic syndrome in women with previous gestational diabetes. Diabetes Metab Res Rev 23:135-140

27. Catalano PM, Hoegh M, Minium J et al (2006) Adiponectin in human pregnancy: implications for regulation of glucose and lipid metabolism. Diabetologia 49:1677-1685

28. Kharroubi I, Rasschaert J, Eizirik DL, Cnop M (2003) Expression of adiponectin receptors in pancreatic beta cells. Biochem Biophys Res Commun 312:1118-1122

29. Staiger K, Stefan N, Staiger H et al (2005) Adiponectin is functionally active in human islets but does not affect insulin secretory function or beta cell lipoapoptosis. J Clin Endocrinol Metab 90:6707-6713

30. Okamoto M, Ohara-Imaizumi M, Kubota N et al (2008) Adiponectin induces insulin secretion in vitro and in vivo at a low glucose concentration. Diabetologia 51:827-835

31. Retnakaran R, Hanley AJ, Raif N et al (2005) Adiponectin and beta cell dysfunction in gestational diabetes: pathophysiological implications. Diabetologia 48:993-1001

32. Bacha F, Saad R, Gungor N, Arslanian SA (2004) Adiponectin in youth: relationship to visceral adiposity, insulin sensitivity, and beta cell function. Diabetes Care 27:547-552
33. Guldstrand M, Ahrén B, Adamson U (2004) Improved beta cell function after standardized weight reduction in severely obese subjects. Am J Physiol Endocrinol Metab 284:E557E565

34. Musso G, Gambino R, Biroli G et al (2005) Hypoadiponectinemia predicts the severity of hepatic fibrosis and pancreatic beta cell dysfunction in nondiabetic nonobese patients with nonalcoholic steatohepatitis. Am J Gastroenterol 100:24382446

35. Koebnick C, Roberts CK, Shaibi GQ et al (2008) Adiponectin and leptin are independently associated with insulin sensitivity, but not with insulin secretion or beta cell function in overweight Hispanic adolescents. Horm Metab Res 40:708-712

36. Clark HD, Graham ID, Karovitch A, Keely EJ (2009) Do postal reminders increase postpartum screening of diabetes mellitus in women with gestational diabetes mellitus? A randomized controlled trial. Am J Obstet Gynecol 200(634): e1-e7

37. Buchanan TA, Xiang AH, Peters RK et al (2002) Preservation of pancreatic beta cell function and prevention of type 2 diabetes by pharmacological treatment of insulin resistance in high-risk Hispanic women. Diabetes 51:2796-2803

38. Yu JG, Javorschi S, Hevener AL et al (2002) The effect of thiazolidinediones on plasma adiponectin levels in normal, obese and type 2 diabetic subjects. Diabetes 51:2968-2974 